\title{
Stationary flow, first passage times, and macroscopic Fick's first diffusion law: Application to flow enhancement by particle trapping
}

\author{
Wolfgang R. Bauer ${ }^{\mathrm{a})}$ \\ Medizinische Universitätsklinik I, Universität Würzburg, Josef Schneiderstrasse 2, D-97080 Würzburg \\ Walter Nadler \\ Fakultät für Physik, Bergische Universität, Gaußstrasse 20, D-42097 Wuppertal, Germany \\ and NIC clo Forschungszentrum Jülich, D-52725 Jülich, Germany
}

(Received 4 March 2005; accepted 2 May 2005; published online 29 June 2005)

\begin{abstract}
A generalized macroscopic Fick's first diffusion law is derived which describes steady-state particle flow between two baths explicitly as a function of the concentration gradient, acting as the thermodynamic driving force, times a functional of the first passage time. The latter is shown to be the ratio of the number of particles trapped between the baths and the first passage time. Particle trapping is shown to be a powerful mechanism by which flow can be enhanced. This is analyzed for two examples: a potential and an entropy trap. () 2005 American Institute of Physics. [DOI: $10.1063 / 1.1940056]$
\end{abstract}

\section{INTRODUCTION}

Diffusive transport driven by concentration gradients plays an important role in many fields, ranging from physical to biological systems. Many scenarios may be reduced to the generic model of steady-state diffusion transport between two baths of different particle concentrations, i.e., the concentration gradient acts as the driving force for particle transport. Diffusion transport can be characterized by global parameters as concentration $c$, flow $J$, and microscopic parameters which are related to a single particle, e.g., the local mobility as is given by the diffusion coefficient. One important equation, combining both is Fick's first law of diffusion, which in the one-dimensional (1D) case under the assumption of homogeneous diffusion properties ${ }^{1}$ takes the form

$$
J=\frac{D}{L}\left(c_{1}-c_{2}\right)
$$

Here $D$ is the diffusion coefficient, and $L$ is the length of the path connecting the baths with concentrations $c_{1}$ and $c_{2}$.

In Eq. (1) flow is related to the gradient of concentrations, which acts as the thermodynamic driving force between the baths, the diffusion coefficient as a microscopic local (environmental) parameter, and the length as a geometrical quantity of the space between the baths. The question arises whether a macroscopic diffusion law as given by Eq. (1) may be generalized to arbitrary diffusion processes between two baths. Intuitively one would think that flow should be proportional to the thermodynamic driving force and some microscopic parameter, describing the average fate of a particle between the baths. ${ }^{2}$ A parameter quantifying this history of a particle is the mean first passage time in which the mean time from the particle stays in between the baths, from delivery until absorption.

\footnotetext{
${ }^{\text {a)} E l e c t r o n i c ~ m a i l: ~ w . b a u e r @ m e d i z i n . u n i-w u e r z b u r g . d e ~}$
}

At a first glance one would think that knowledge of the first passage time and particle concentration in the source is sufficient to determine the particle flow to the absorber. We consider bath 1 as the source with $c_{1}=c$ and bath 2 as a pure absorber, i.e., $c_{2}=0$. The number of particles expelled from bath 1 is proportional to $c_{1}$ i.e., particle flow $J$ from bath 1 to bath 2 , as well. The inverse first passage time $\tau^{-1}$ is the transition rate from bath 1 to 2, i.e., proportional to $J$, and combining both results in

$$
J \sim c \frac{1}{\tau}
$$

Similarly one obtains particle flow from bath 2 to bath 1 by interchanging source and absorber and substraction of flows provides the net flow.

This consideration implies that the main parameters determining flow are the concentration gradient and the first passage time. However, this simplistic view does not consider the following points: the proportionality factor in Eq. (2) remains to be determined in either direction of transport. Furthermore the definition of a first passage time is problematic when a bath acts as a particle source, since particles may reenter.

Hardt $^{3}$ considered unidirectional flow between a source, expelling particles at constant rate and an absorber. In between source and absorber free diffusion was assumed. It was shown that

$$
J=\frac{N}{\tau}
$$

where $N$ denotes the number of particles trapped in the system between source and absorber. However, there are several constraints: Hardt assumed that particles are uniformly expelled and that there is free diffusion between source and absorber. Furthermore $N$ is a macroscopic parameter, i.e., when we try to formulate a diffusion law which is solely based on the thermodynamic driving force, and the history of 
a particle, the dependence of $N$ on the concentration gradient and first passage time remains to be determined.

It is the aim of this paper to present a rigorous generalization of Hardt's approach for the two-bath scenario. Based on this we will derive a general macroscopic Fick's first diffusion law which is solely based on the first passage time, or more precisely a functional of the first passage time, and the concentration gradient. This functional of the first passage time provides the missing proportionality factor in relation (2). For didactically reasons we derive the relations for the 1D case, the more general formulation for diffusion between baths in $n$ dimensions is considered in the Appendix.

This general diffusion law will have a more complex form than what the simplistic relation (2) suggests. This has implications when considering mechanisms which modulate flow between the baths. Based on relation (2), focus in the past mainly laid on alterations of the first passage time, e.g., shortening of the first passage time enhances flow, and, vice versa, its prolongation reduces flow. Our result demonstrates that besides the first passage time, particle trapping is another powerful mechanism to enhance flow. Particle trapping may be achieved by an attractive potential or an entropy trap. Both examples are considered in this paper. Particle trapping during diffusion transport was discussed for transport across transmembrane channels, with a potential well inside the channel, ${ }^{4}$ and found for incorporation of antibiotics through bacterial pores. ${ }^{5}$ The mechanism of particle trapping by an entropy trap is most likely realized in some enzymatic reactions. On the other side, one could think of constructing entropy traps in nanotechnological applications for, e.g., the development of molecular motors.

\section{FIRST PASSAGE TIME AND MACROSCOPIC STEADY-STATE PARAMETERS}

In this section we will relate the macroscopic steadystate parameters particle number/density and flow to the first passage time, which provides information about the history of particles.

We consider $1 \mathrm{D}$ diffusion along a path of length $L$, however, this may be generalized to $n \mathrm{D}$ diffusion. The beginning of the path is located at $x=0$, where it is adjacent to a bath with particle concentration $c$ and at its end at $x=L$ particles are instantaneously absorbed, i.e., there is unidirectional flow from bath 1 to bath 2. ${ }^{6}$ Diffusion dynamics is determined by a Fokker-Planck equation, i.e., for the probability density the following holds:

$$
\partial_{t} \rho(x, t)=\Upsilon \rho(x, t),
$$

with

$$
\Upsilon=\partial_{x} D(x)\left(\partial_{x}-F(x)\right),
$$

with $Y$ as the generator of this process, $D(x)$ denotes the diffusion coefficient, and $F(x)$ is the driving force. ${ }^{7}$

In the steady state we have $\partial_{t} \rho \equiv 0$, i.e., particle flow $J$ is constant in time. All macroscopic steady-state parameters are obtained from the steady-state density $\rho_{s}(x)$. This steadystate density would remain unchanged when we replace the bath by a pure flow source at $x=0$ with flow $J$ and introduce reflecting boundary conditions at $x=0$ for particles which are on the path. Though, from a macroscopic point of view the steady-state parameters are identical, the microscopic point of view demonstrates an entire difference between both setups. Whereas there is a particle exchange between bath 1 and the path in the first setup, the second only allows a unidirectional inflow of particles from bath 1 . The particles which have entered the path may only leave the path at its end $x$ $=L$, i.e., there is no reabsorption by bath 1 . This new setup allows the introduction of microscopic parameters which are related to the history of particles on the path. For example, we are now able to define the first passage time as the mean time between particle delivery from bath 1 until absorption by bath 2 . It has to be stressed that this definition of a first passage time as a mean residence time cannot be readily transferred to the original setup with potential reabsorption of particles by bath 1 . The dynamics in the setup with reflecting boundary conditions at $x=0$ is not anymore determined by Eq. (5) but by

$$
\partial_{t} \rho(x, t)=\Lambda \rho(x, t)+J \delta(x) .
$$

The term $J \delta(x)$ describes the inflow of particles at the origin $x=0$. The generator $\Lambda$ is identical to the original generator $Y$ except that it considers reflecting boundary conditions at $x$ $=0$. As mentioned above the steady-state density derived from Eq. (4) is identical to that of Eq. (6), i.e.,

$$
\rho_{s}(x)=-J \Lambda^{-1} \delta(x) .
$$

The term $-\Lambda^{-1} \delta(x)$ in Eq. (7) may be rewritten in the form

$$
\begin{aligned}
-\Lambda^{-1} \delta(x) & =\int_{0}^{\infty} d t[\exp (\Lambda t)]_{x, 0}-\lim _{t \rightarrow \infty} \Lambda^{-1}[\exp (\Lambda t)]_{x, 0} \\
& =\int_{0}^{\infty} d t[\exp (\Lambda t)]_{x, 0},
\end{aligned}
$$

where $[\exp (\Lambda t)]_{x, x^{\prime}}$ is Green's function or propagator of the process generated by $\Lambda$, i.e., the probability density to find a particle at time $t$ at position $x$ when it has started at position $x^{\prime}$. The absorptive boundary condition at $x=L$ and the finite diffusion interval implies that all eigenvalues of $\Lambda$ are negative with an upper nonvanishing boundary. Hence, $\Lambda^{-1}[\exp (\Lambda t)]_{x, 0}$ vanishes for $t \rightarrow \infty$ giving Eq. (8).

The probability to find a particle within the interval $[0, L]$ when it has started at the origin is obtained from integration of Green's function over the interval $[0, L]$, i.e.,

$$
p_{[0, L]}(t)=\int_{0}^{L} d x[\exp (\Lambda t)]_{x, 0} .
$$

The number of particles present in the interval in the steady state is obtained from integration of the steady-state density of Eq. (7). Together with Eqs. (8) and (9) this results in

$$
N_{[0, L]}=\int_{0}^{L} d x \rho_{s}(x)=J \int_{0}^{\infty} d t p_{[0, L]}(t)=J \tau_{[0, L]} .
$$

Here 


$$
\tau_{[0, L]}=\int_{0}^{\infty} d t p_{[0, L]}(t)
$$

is the mean first passage time of a particle having started at the origin $x=0$. From a microscopic viewpoint, this is the mean time that a particle stays within the interval, from source delivery until absorption.

The considerations above can be generalized from the interval $[0, L]$ to subintervals $[\xi, L]$. A flow source is introduced at position $\xi$ with flow $J$. Reflective boundary conditions at $x=\xi$ are assumed for particles within $[\xi, L]$, i.e.,

$$
N_{[\xi, L]}=J \tau_{[\xi, L]},
$$

with $N_{[\xi, L]}$ as the number of particles within the interval $[\xi, L]$ and $\tau_{[\xi, L]}$ as the first passage time of a particle having started at $x=\xi$.

Since

$$
\rho_{s}(x)=-\partial_{x} N_{[x, L]}=\partial_{L-x} N_{[x, L]},
$$

we obtain the fundamental equation

$$
\rho_{s}(x)=J \partial_{L-x} \tau_{[x, L]},
$$

which relates the steady-state density to flow and the derivative of the first passage time. We referred the particle number and first passage time to the length of the interval $L-x$ rather than the position $x$ to make the formula conform with that in $n$ dimensions (see Appendix). Especially for $x=0$ we obtain

$$
c=\left.J \partial_{L-x} \tau_{[x, L]}\right|_{x=0} .
$$

As shown in the Appendix transport along the finite interval $[0, L]$ can be generalized to that from a bath through an $n$-dimensional closed domain $U$, where bath 1 is adjacent to some boundary $\partial_{1} U$ and bath 2 to $\partial_{2} U$. The relations between particle number within the domain $N_{U}$, first passage time $\tau_{U}$, and flow then generalize from Eqs. (10) and (15) to

$$
\begin{aligned}
& N_{U}=J \tau_{U}, \\
& c=\left.J \partial_{V} \tau_{x}\right|_{x \in \partial_{1} U},
\end{aligned}
$$

where $V$ is the volume of $U$ and $x$ is adjacent to bath 1, i.e., $x \in \partial_{1} U$.

\section{GENERAL FORMULATION OF A MACROSCOPIC FICK'S FIRST DIFFUSION LAW}

Fick's diffusion law states that in the steady state there is a linear relationship between particle flow and concentration gradient between two baths. Based on the previous considerations we will formulate a general expression of Fick's diffusion law, derived from scaling properties.

First, we will focus on the scenario in which one bath acts as a source (bath 1) and the second as an absorber, i.e., particle concentration at the boundary adjacent to the latter bath vanishes (bath 2). From Eq. (17) it then directly follows the dependence of flow on particle concentration in the bath and the derivative of the first passage time,

$$
J=\left(\partial_{V} \tau_{x}\right)^{-1} c,
$$

where $x$ is an arbitrary point of the boundary adjacent to bath 1 . The first passage time $\tau_{x}$ and its derivative $\partial_{V} \tau_{x}$ are microscopic quantities, i.e., they refer to the average fate of a single particle starting at the boundary adjacent to the source bath. As long as one assumes that particles under consideration diffuse independently, the first passage time and its derivative are independent of concentrations, i.e., $c$. Hence, Eq. (18) provides a linear relation between particle flow and particle concentration of the source,

$$
J \sim c .
$$

Note that this relation is not as trivial as it appears at a first glance. Equation (18) explicitly relates flow and particle concentration within the bath to the microscopic parameter "derivative of the first passage time" $\partial_{V} \tau_{x}$. Only the independence of the latter on particle concentration implies linear Fick's diffusion law and vice versa.

Since the first passage time is independent of the bath concentration, whereas flow depends linearly on it, Eq. (16) implies that the number of particles also depends linearly on the bath concentration, i.e.,

$$
N_{U} \sim c .
$$

The last equations suggest to introduce the specific number of particles $n_{U}$ which is the number of particles in the domain normalized to the bath concentration,

$$
n_{U}=\frac{N_{U}}{c} .
$$

Equation (16) then implies another formulation of Fick's diffusion law,

$$
J=\frac{n_{U}}{\tau} c,
$$

where the interrelation between specific particle number, first passage time, and its derivative is given by [see Eq. (18)]

$$
\frac{n_{U}}{\tau}=\left(\partial_{V} \tau_{x}\right)^{-1} .
$$

Note that the above equation directly provides a simple interpretation of the derivative of the first passage time.

Fick's equation (22) may be readily transferred to the scenario of two baths releasing and absorbing particles to and from the domain $U$. Since the particles are noninteracting one bath may be considered as the source the other as the absorber and vice versa. In the case of two baths with concentrations $c_{1}$ and $c_{2}$ the flow at the boundaries is

$$
J=\left(\frac{n_{1,2}}{\tau_{1,2}}\right) c_{1}-\left(\frac{n_{2,1}}{\tau_{2,1}}\right) c_{2},
$$

where $n_{i, j} \tau_{i, j}$ denote the specific number of particles and first passage time when bath $i$ is referred to as the source and $j$ as the absorber.

Equation (24) is a very general formulation of Fick's diffusion law, which makes no constraints on the driving forces within the domain. We will now restrict our consider- 
ations to systems which may reach an equilibrium state for an appropriate choice of bath concentrations. Hence, for every concentration $c_{1}$ there exists a concentration $c_{2}$ in bath 2 , providing a steady-state concentration $\rho_{e}(x)$ for which microreversibility holds. Microreversibility implies that the principle of detailed balance is valid, i.e., local flows vanish,

$$
j_{e}(x)=-D\left(\partial_{x}-F(x)\right) \rho_{e}(x) \equiv 0 .
$$

This suggests to introduce a potential $\Phi(x)$ derived from the equilibrium concentration $\rho_{e}(x)$ as

$$
\Phi(x)-\Phi\left(x_{0}\right)=-\ln \left(\rho_{e}(x) / \rho_{e}\left(x_{0}\right)\right),
$$

where $x_{0}$ is some arbitrary point within the domain which defines the offset of the potential. Combination of the latter equations implies

$$
F(x)=-\partial_{x} \Phi(x),
$$

i.e., the existence of detailed balance implies a conservative force field and vice versa. ${ }^{8}$

A constant $\rho_{e}$ at the boundaries adjacent to the baths implies according to Eq. (26) that these boundaries are equipotential lines, the value of which we will refer to as $\Phi_{1}$ and $\Phi_{2}$. They fulfill the relation

$$
e^{\Phi_{1}-\Phi_{2}}=c_{2, e} / c_{1} .
$$

Since flow vanishes in the equilibrium we obtain from Eq. (24)

$$
\frac{n_{1,2}}{\tau_{1,2}} e^{-\Phi_{1}}=\frac{n_{2,1}}{\tau_{2,1}} e^{-\Phi_{2}}
$$

i.e., the term

$$
\vartheta=\frac{n_{i, j}}{\tau_{i, j}} e^{-\Phi_{i}}, \quad i, j=1,2, \quad i \neq j
$$

may be interpreted as the diffusive conductivity which is independent of the direction of transport between the baths. Hence, introducing the chemical potentials

$$
\mu_{i}=\Phi_{i}+\ln c_{i},
$$

we can formulate Fick's diffusion law as

$$
J=\vartheta\left(e^{\mu_{1}}-e^{\mu_{2}}\right) .
$$

And, when both baths are on the same potential level, i.e., $\Phi_{1}=\Phi_{2}$, we have

$$
\vartheta=\frac{n_{1,2}}{\tau_{1,2}}=\frac{n_{2,1}}{\tau_{2,1}},
$$

where, without loss of generality, the bath potential was normalized to zero. Fick's diffusion law then reads

$$
J=\vartheta\left(c_{1}-c_{2}\right)=\frac{n}{\tau}\left(c_{1}-c_{2}\right),
$$

where we omitted the indices to demonstrate that either direction may be chosen for the determination of the conductivity. Note that the equivalence of the conductivities in either direction of transport between the baths does not imply that of the respective specific particles number or first pas- sage time, i.e., in general we have do not have $n_{1,2}=n_{2,1}$ or $\tau_{1,2}=\tau_{2,1}$.

In summary we demonstrated in this section that particle flow between two baths one acting as a source the other as a pure absorber may be solely related to the microscopic parameter first passage time (more precisely its derivative) and the concentration as a driving force. The rather abstract expression for the conductivity $\left(\partial_{V} \tau_{U_{x}}\right)^{-1}$ is equivalent to the ratio of the specific particle number and first passage time. Linear superposition provides a formulation of Fick's diffusion law for arbitrary concentrations of the baths [Eq. (24)]. When we restrict to systems in which detailed balance is existent, the diffusive conductivities of particle transport are independent of the direction of transport, i.e., from bath 1 to 2 and vice versa [Eqs. (29) and (30)]. For this case we are able to write Fick's diffusion law in Eqs. (32) and (34) in the requested form of Eq. (1).

\section{APPLICATIONS: ENHANCEMENT OF FLOW BY PARTICLE TRAPPING}

\section{A. Potential trap}

We consider 1D diffusion along a path of length $L$ between a bath acting as a source located at $x=0$ and an absorbing bath at $x=L$. On their way particles are affected by some drift force $F(x)$ [see Eq. (5)] resulting from a potential $\Phi$, i.e.,

$$
F(x)=-\partial_{x} \Phi(x) .
$$

We only focus on the concentration gradient as the driving force of particles between the baths, i.e., the potential is assumed to vanish at the edges of the path,

$$
\Phi(0)=\Phi(L)=0 .
$$

The determination of the first passage time in 1D diffusive processes is well established. ${ }^{9,10}$ When $\tau_{[x, L]}$ denotes the first passage time of a particle starting at position $x$ within the path, with reflecting boundary conditions at $x$ and absorptive at $L$, this parameter is obtained as

$$
\tau_{[x, L]}=D^{-1} \int_{x}^{L} \int_{x}^{L} d \eta d \xi \theta(\eta-\xi) e^{\Phi(\eta)} e^{-\Phi(\xi)},
$$

where $\theta(\zeta)$ denotes the Heaviside function, i.e., $\theta(\zeta) \equiv 0$ for $\zeta<0$ and $\theta(\zeta) \equiv 1$ for $\zeta \geqslant 0$.

The specific particle number within the interval $[0, L]$ is obtained from Eqs. (23) and (37) as

$$
n=\frac{\int_{0}^{L} \int_{0}^{L} d \eta d \xi \theta(\eta-\xi) e^{\Phi(\eta)} e^{-\Phi(\xi)}}{\int_{0}^{L} d \eta e^{\Phi(\eta)}}
$$

The latter two equations demonstrate that the effect of the potential $\Phi$ on the specific particle number differs from that on the corresponding first passage time $\tau_{[0, L]}$ by the factor 


$$
\left(\int_{0}^{L} d \eta e^{\Phi(\eta)}\right)^{-1}=\left(L\left\langle e^{\Phi(\eta)}\right\rangle\right)^{-1},
$$

where the angular brackets \langle\rangle denote the spatial average over the interval $[0, L]$. This implies for the flow [see Eq. (34)]

$$
J=J_{0}\left\langle e^{\Phi(x)}\right\rangle^{-1},
$$

where

$$
J_{0}=c D / L
$$

is the flow for a vanishing potential, $\Phi(x) \equiv 0$. Equation (40) demonstrates that the factor $\left\langle e^{\Phi(x)}\right\rangle^{-1}$ may be interpreted as some kind of averaged Arrhenius factor, relating flow to the magnitude of the potential. Furthermore this equation shows that for a pure potential barrier $\Phi(x)>0$ particle flow is decreased when related to that of a vanishing potential. Conversely a pure potential trap $\Phi(x)<0$ always increases flow. In both cases the effect of the potential on the specific particle number is responsible for this flow behavior as Eqs. (37) and (38) demonstrate.

We will now discuss the special case of a symmetrical potential, i.e., $\Phi(x)=\Phi(L-x)$. Exploiting this symmetry in Eq. (37) provides for the first passage time $\tau=\tau_{[0, L]}$,

$$
\begin{aligned}
\tau & =D^{-1} \frac{1}{2} \int_{0}^{L} \int_{0}^{L} d \eta d \xi \cosh (\Phi(\eta)-\Phi(\xi)) \\
& =\frac{L^{2}}{2 D}\left\langle e^{\Phi(x)}\right\rangle\left\langle e^{-\Phi(x)}\right\rangle=\tau_{0}\left\langle e^{\Phi(x)}\right\rangle\left\langle e^{-\Phi(x)}\right\rangle,
\end{aligned}
$$

where $\tau_{0}$ is the first passage time for a vanishing potential. And from Eq. (38) follows

$$
n=n_{0}\left\langle e^{-\Phi(x)}\right\rangle,
$$

where

$$
n_{0}=L / 2
$$

denotes the specific particle number for a vanishing potential.

The symmetry of the first passage time in Eq. (42) $\tau(\Phi)=\tau(-\Phi)$ demonstrates the well-known fact that a symmetrical trap has the same first passage as its corresponding barrier. Since $\cosh (\Phi(\eta)-\Phi(\xi)) \geqslant 1$, Eq. (42) implies for a symmetrical potential

$$
\tau \geqslant \tau_{0},
$$

i.e., the potential, either trap or barrier prolongs the first passage time when related to the system with vanishing potential.

On the other hand, the effect of the potential on the specific number of particles within the path is not invariant under the transformation $\Phi \rightarrow-\Phi$ as Eq. (43) shows. A barrier $(\Phi(x)>0)$ reduces the specific particle number, i.e., the effects of the potential on the first passage time and specific particle number are concordant in reducing particle flow.

In the case of a potential trap $(\Phi<0)$, the particle number is increased when related to a vanishing potential. Hence, two opposing effects affect particle flow. However, the effect of the trap on prolongation of the first passage time is infe- rior to that on the specific particle number, since the factor $\left\langle e^{\Phi(x)}\right\rangle$ in Eq. (42) is smaller than 1. Hence, the potential trap increases flow as Eq. (40) demonstrates.

A very simple realization of a symmetrical potential is a rectangular potential, located symmetrically around $L / 2$, i.e., $\Phi(x)=\Phi_{0}$ for $(1-w) L / 2<x<(1+w) L / 2$, and elsewhere zero. Here the parameter $w(0 \leqslant w \leqslant 1)$ denotes the relative width of the potential [Fig. 1(a)]. Then the first passage time is

$$
\tau=\tau_{0}\left(1+2 w(1-w)\left(\cosh \left(\Phi_{0}\right)-1\right)\right) .
$$

Relative particle number and, hence, flow are

$$
\begin{aligned}
& n=n_{0}\left(1+w\left(e^{-\Phi_{0}}-1\right)\right), \\
& J=J_{0}\left(1+w\left(e^{\Phi_{0}}-1\right)\right)^{-1} .
\end{aligned}
$$

Figures 1(b)-1(d) show the course of these parameters as a function of the level $\Phi_{0}$. When we study a trap $\left(\Phi_{0}<0\right)$, it is obvious that despite an increase of the first passage time [Fig. 1(b)], flow increases due to particle trapping within the potential [Figs. 1(c) and 1(d)]. This very simple example demonstrates the power of a potential trap to increase concentration gradient driven particle flow despite an increase of the first passage time.

We discuss now some special cases for the rectangular potential. First, we assume that the relation

$$
w(1-w) e^{\left|\Phi_{0}\right|} \gg 1
$$

holds, i.e., the potential has a sufficient depth/height related to an intermediate width. Then we obtain from Eq. (46) for the first passage time

$$
\tau=\tau_{0} w(1-w) e^{\left|\Phi_{0}\right|} .
$$

Hence, the first passage time increases exponentially with the absolute value of the potential level [Fig. 1(b)] and depends on the factor $w(1-w)$, which defines the geometry of the trap. Equation (50) is Kramer's formula ${ }^{11}$ giving the escape time for barrier crossing, which is adapted to a rectangular potential. Note that in the case of a symmetrical potential the first passage time to pass a trap is that of crossing the corresponding barrier.

When the rectangular potential acts as a barrier $\left(\Phi_{0}\right.$ $>0$ ) we derive for the specific particle number and flow

$$
\begin{aligned}
& n=n_{0}(1-w), \\
& J=J_{0} w e^{-\Phi_{0},}
\end{aligned}
$$

i.e., the specific particle number is identical to that for free diffusion along a reduced path length $L^{\prime}=L(1-w)$ [Fig. 1(c)]. The flow follows the classical Arrhenius relation [Fig. $1(\mathrm{~d})]$.

When the potential acts as a particle trap, i.e., $\Phi_{0}<0$, we obtain

$$
\begin{aligned}
& n=n_{0} w e^{\left|\Phi_{0}\right|}, \\
& J=J_{0} /(1-w),
\end{aligned}
$$

i.e., the specific particle number increases as the first passage time exponentially with the depth of the trap [Fig. 1(c)], and, 


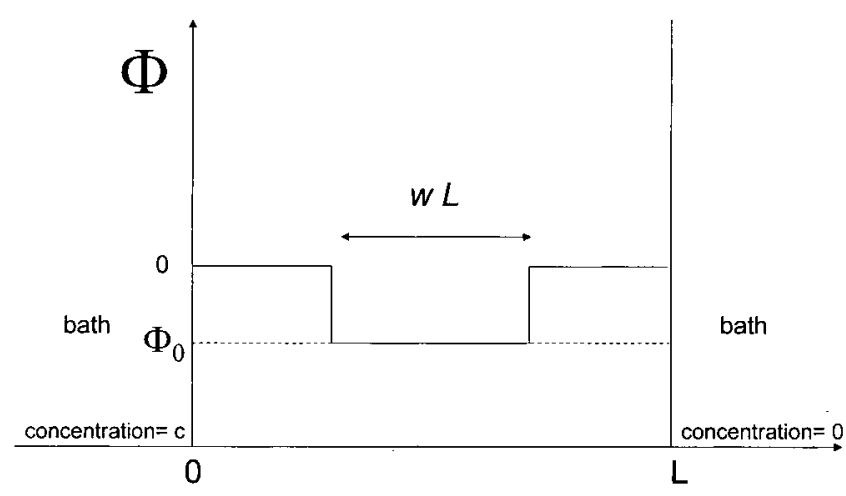

(a)

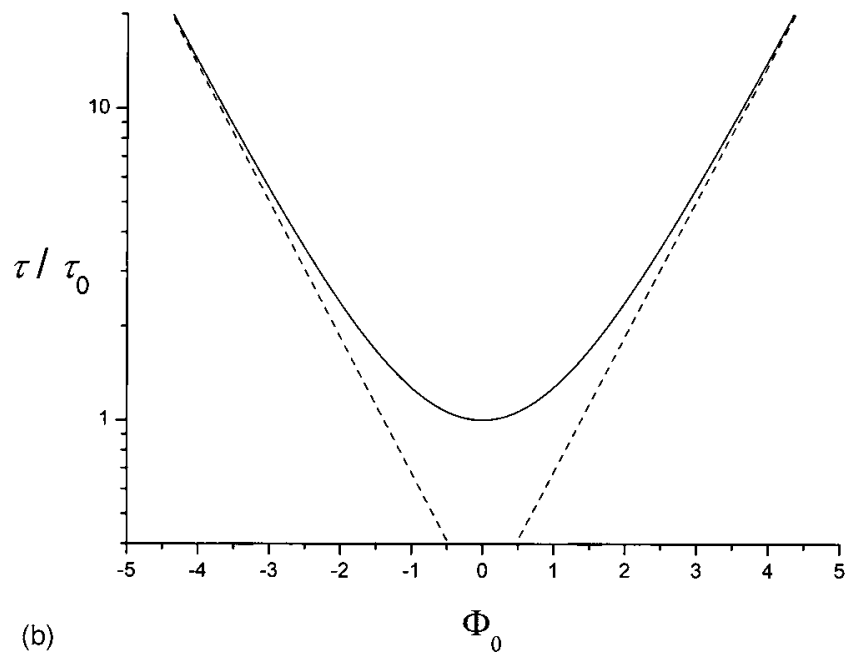

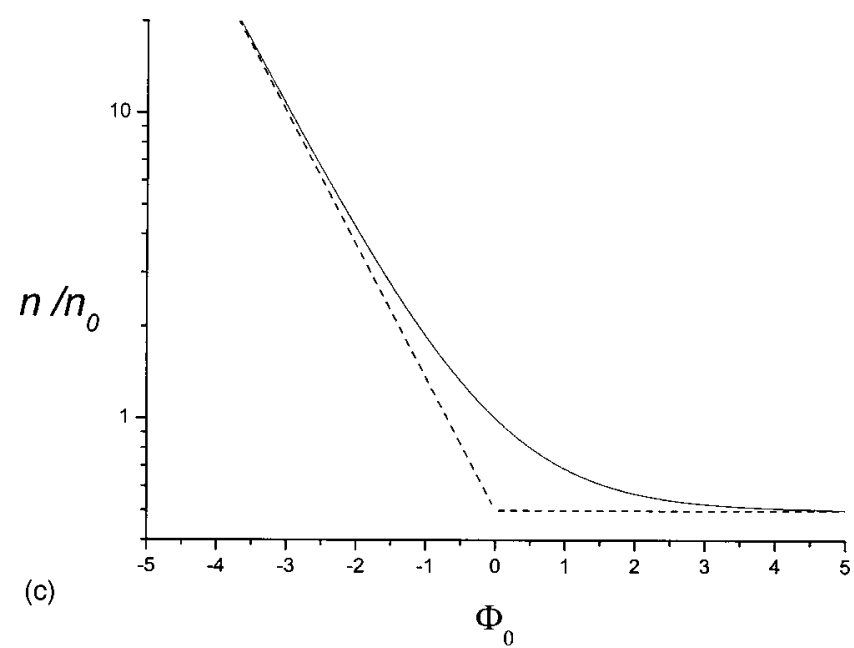

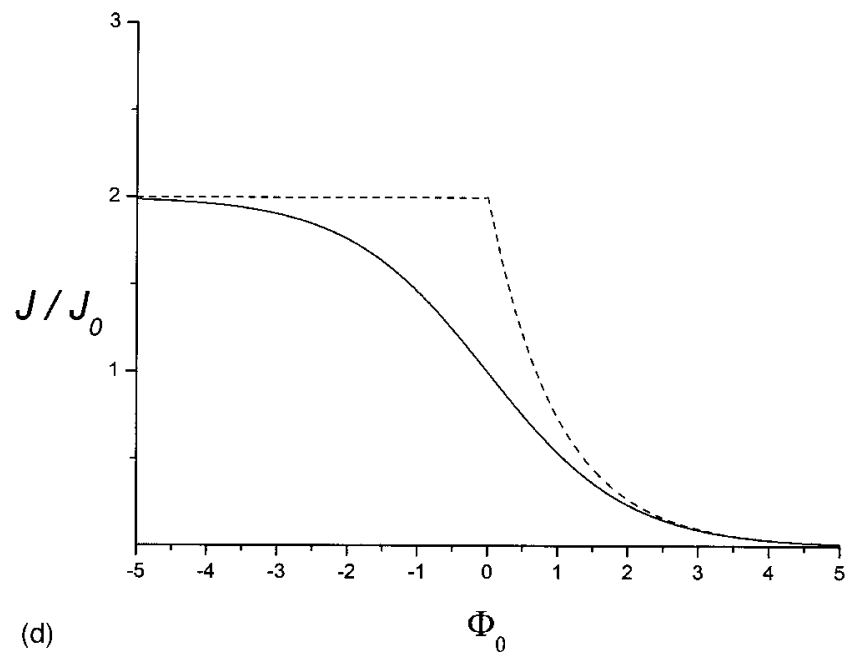

FIG. 1. Schematic of a rectangular symmetrical potential (a) along the path of length $L$. The first passage time (b), specific particle number (c), and flow (d) are shown as functions [see Eqs. (46)-(48)] of the level $\Phi_{0}$ of this potential (solid lines). A positive $\Phi_{0}$ acts as a barrier, a negative as a trap. The relative width of the potential was assumed to be $w=1 / 2$. The dashed lines are approximations when the absolute value of the potential satisfies $e^{\left|\Phi_{0}\right|} w(\mathbf{1}-w) \gg 1($ see text).

hence, flow increases asymptotically to the above limiting value [Fig. 1(d)]. This value is identical to free-diffusive flow along a path of the reduced length $L^{\prime}=(1-w) L$, i.e., the trap apparently shortens the the diffusion length.

Another limiting case is when the potential fills the whole path $w \rightarrow 1$. Then, the first passage time is that of a vanishing potential, i.e., one has

$$
\tau(w \rightarrow 1)=\tau_{0} .
$$

The specific particle number and hence flow are then

$$
\begin{aligned}
& n(w \rightarrow 1)=n_{0} e^{-\Phi_{0}}, \\
& J(w \rightarrow 1)=J_{0} e^{-\Phi_{0}},
\end{aligned}
$$

i.e., modulations of flow by the potential solely depend on how this potential affects the particle number since the first passage time is not affected.

\section{B. Entropy trap}

As in the case of the potential trap we study 1D diffusion transport along a path of length $L$ between two baths. An increase of the entropy on the path is realized by allowing the particles to have here additional degrees of freedom (Fig. 2 ). The state of a particle is characterized by its position $x$ and an additional stochastic variable $i$. This, for example, may be realized by additional spatial degrees of freedom, or a reaction coordinate which allows particles to undergo chemical transitions. Trapping is realized by the constraint that the baths shall have only access to one state of this additional variable which is denoted as $j$.

For simplicity we assume within the trap homogeneous, free diffusion in the $x$ direction, which is independent of the additional state variable $i$, except at the boundaries, $x=0, L$. Here are reflecting boundary conditions for $i \neq j$, whereas the baths have free access to the state $i=j$. A stationary Markovian dynamics of the variable $i$ is assumed, which is determined by the transition rate matrix $\mathbf{R}=\left(r_{i_{1}, i_{2}}\right)$, i.e., $r_{i_{1}, i_{2}}$ is the transition rate from state $i_{2} \rightarrow i_{1}$. On the path we consider the particle density with respect to the stochastic state variable,

$$
\boldsymbol{\rho}=\left(\rho_{i}\right),
$$

i.e., the particle density is the sum over these restricted densities, 


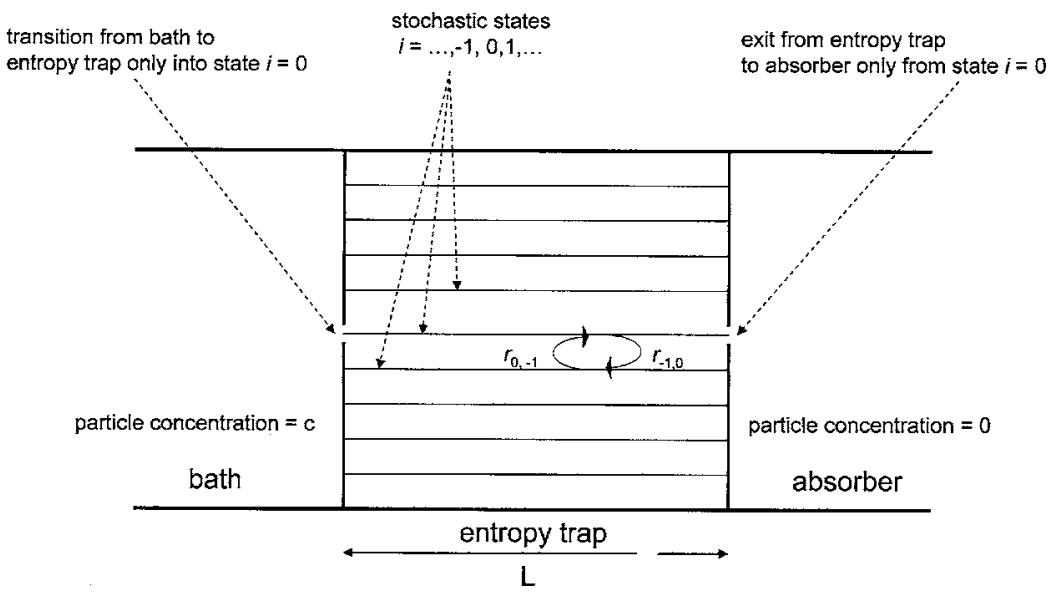

FIG. 2. Schematic of an entropy trap. The system is adjacent to two baths, one with concentration $c$ and the other acting as a pure absorber, i.e., with vanishing particle concentration. Within the trap particles have an additional degree of freedom, characterized by the state variable $i$. The baths have access only to one stochastic state, which in this example is $i=0$. Within the trap, the state variable undergoes stochastic transition $i_{1} \rightarrow i_{2}$ with the rates $r_{i_{2}, i_{1}}$. In the schematic the transition -1 $\rightarrow 0$ and vice versa is shown.

$$
\rho(x)=\sum_{i} \rho_{i}(x)
$$

For the evolution of this system one has to consider that within an infinitesimal small time interval $d t$ the dynamics of the composed variable $(x, i)$ is a linear superposition of diffusion and state transition dynamics. Hence, the evolution of the particle density follows

$$
\partial_{t} \boldsymbol{\rho}(x, t)=\left(\mathbf{R}+D \partial_{x}^{2}\right) \boldsymbol{\rho}(x, t),
$$

i.e., the steady-state density fulfills

$$
\left(\mathbf{R}+D \partial_{x}^{2}\right) \boldsymbol{\rho}_{s}(x)=0 .
$$

We have now introduced the system. First, we consider how it works under equilibrium conditions to make its ability to act as an entropy trap evident. Equilibrium is realized when the two baths have identical particle concentrations $c$. When $p_{0, i}$ denotes the equilibrium probability distribution of $\mathbf{R}$, the equilibrium particle density on the path is enhanced, when compared to that of the baths, i.e.,

$$
\rho_{e}(x) \equiv c \frac{1}{p_{0, j}} .
$$

Hence, one can define the entropy of the trap as

$$
S=\ln \left(\frac{\rho_{e}}{c}\right)=-\ln p_{0 . j}
$$

We will now focus on the steady state for the generic case when one bath acts as a source with concentration $c$ and the other as a pure absorber. This implies the boundary conditions

$$
\begin{aligned}
& \rho_{j}(0)=c, \\
& \rho_{j}(L)=0,
\end{aligned}
$$

since only the stochastic state $j$ has access to the baths. The other boundary conditions follow from the reflecting boundary conditions for stochastic states $i \neq j$. In these state flows at $x=0$ and $x=L$ vanish. In the state $j$, inflow and outflow are equivalent. Formally these flow-related boundary conditions are summarized in

$$
\partial_{x} \boldsymbol{\rho}_{s}(0)=\partial_{x} \boldsymbol{\rho}_{s}(L)=-J / D \mathbf{e}_{j},
$$

where $\mathbf{e}_{j}=(0, \ldots, 1, \ldots, 0)^{t}$ is the $j$ th unit vector in the stochastic state space.

Determination of the specific particle number, first passage time, and flow requires some algebra (see Appendix). One obtains

$$
\begin{aligned}
& n=n_{0} e^{S}, \\
& \tau=\tau_{0}\left(\sum_{\nu \neq 0} \mathbf{e}_{j}^{t} \mathbf{r}_{\nu} \mathbf{l}_{\nu}^{t} \mathbf{e}_{j} \frac{\tanh \left(\sqrt{\lambda_{\nu} / D} L / 2\right)}{\sqrt{\lambda_{\nu} / D} L / 2} e^{S}+1\right),
\end{aligned}
$$

and

$$
J=\frac{n}{\tau} c=J_{0} e^{S}\left(\sum_{\nu \neq 0} \mathbf{e}_{j}^{t} \mathbf{r}_{\nu} \mathbf{l}_{\nu}^{t} \mathbf{e}_{j} \frac{\tanh \left(\sqrt{\lambda_{\nu} / D} L / 2\right)}{\sqrt{\lambda_{\nu} / D} L / 2} e^{S}+1\right)^{-1},
$$

where $n_{0}, \tau_{0}$, and $J_{0}$ denote the parameters in the absence of the entropy trap, i.e.,

$$
\begin{aligned}
& n_{0}=L / 2, \\
& \tau_{0}=L^{2} /(2 D), \\
& J_{0}=c D / L .
\end{aligned}
$$

The parameters $-\lambda_{\nu}$ and $\nu \neq 0$ denote the nonvanishing negative eigenvalues of the transition rate matrix $\mathbf{R}$, and $\mathbf{l}_{\nu}$ and $\mathbf{r}_{\nu}$ denote the corresponding left- and right-sided eigenvectors, respectively. The terms $\left(\lambda_{\nu} / D\right)^{1 / 2}$ require some interpretation: the average lifetimes of the of $\nu$ th eigenstate of $\mathbf{R}$ is $\lambda_{\nu}^{-1}$. Hence, the characteristic lengths

$$
l_{\nu}=\left(D / \lambda_{\nu}\right)^{1 / 2}
$$

may be considered as the lengths a particle can diffuse during relaxation of the $\nu$ th eigenstate.

Equation (65) demonstrates that the number of particles increased by the trap is solely related to its inherent entropy [Eq. (62)]. This implies that this increase is independent of the transition dynamics between states of the trap. The first passage time in Eq. (66) is the sum of the first passage time for free-1D diffusion $\tau_{0}=L^{2} / 2 D$ and an entropy-related term. In other words, the first passage time increases with the en- 
tropy. Hence, the parameters, specific particle number and first passage time, both having opposed effects on flow [see Eq. (34)], are prolonged by the entropy barrier. However, these effects are not balanced and the typical course of the hyperbolic tangent function implies

$$
\begin{aligned}
\sum_{\nu \neq 0} \mathbf{e}_{j}^{t} \mathbf{r}_{\nu} l_{\nu}^{t} \mathbf{e}_{j} \frac{\tanh \left(L /\left(2 l_{\nu}\right)\right)}{\left(L /\left(2 l_{\nu}\right)\right)} & <\mathbf{e}_{j}^{t}\left(\sum_{\nu \neq 0} \mathbf{r}_{\nu} \mathbf{l}_{\nu}^{t}\right) \mathbf{e}_{j} \\
& =\mathbf{e}_{j}^{t}\left(\mathbf{i d}-\mathbf{r}_{0} \mathbf{l}_{0}^{t}\right) \mathbf{e}_{j}=1-p_{0, j},
\end{aligned}
$$

where the index 0 refers to the equilibrium parameters. Insertion into Eq. (67) and considering that $p_{0, j}=e^{-S}$ [see Eq. (62)] provides

$$
J>J_{0},
$$

i.e., flow is enhanced by the entropy trap when compared to flow $J_{0}$ in its absence. In summary, the entropy trap increases the first passage time and particle trapping, however, the effect on the latter is dominating which results in an increase of flow.

In this context it is instructive to discuss different dynamic ranges of the transition dynamics. When transition dynamics decreases, i.e., $(\mathbf{R} \rightarrow 0)$, one obtains for the first passage time

$$
\lim _{\mathbf{R} \rightarrow 0, \mathbf{R} \neq 0} \tau(\mathbf{R})=\tau_{0} e^{S},
$$

i.e., the particle number and first passage time increase by the same entropy factor and hence the flow approaches that in the absence of the entropy trap

$$
J \rightarrow J_{0} .
$$

At a first glance these results appear surprising since one would assume that a particle at the beginning of the path, i.e., when it is in the state $j$ should remain in this state for vanishing state fluctuations, i.e., the first passage time should be that without stochastic transitions. Instead one has $\tau(\mathbf{R}$ $\rightarrow 0$ ) $>\tau_{0}$, implying that the residence time of those particles which undergo state transition, and which are responsible for the elevated number of particles, increases when the fluctuation dynamics decreases. The contribution of these particles to the mean residence time is responsible for its prolongation when compared to the first passage time in the absence of the entropy trap.

We now discuss the case of increasing fluctuation dynamics, i.e., the eigenvalues $\lambda_{\nu}$ of $\mathbf{R}$ increase and the characteristic lengths $l_{\nu}$ [see Eq. (69)] decrease. The course of the hyperbolic tangent function implies that already for moderate smaller characteristic lengths than the path length one has $\tanh \left(L /\left(2 l_{\nu}\right)\right) \approx 1$. This implies that

$$
\sum_{\nu \neq 0} \mathbf{e}_{j}^{t} \mathbf{r}_{\nu} \mathbf{l}_{\nu}^{t} \mathbf{e}_{j} \frac{\tanh \left(L /\left(2 l_{\nu}\right)\right)}{\left(L /\left(2 l_{\nu}\right)\right)} \approx \frac{2}{L} \sum_{\nu \neq 0} \mathbf{e}_{j}^{t} \mathbf{r}_{\nu} \mathbf{l}_{\nu}^{t} \mathbf{e}_{j} l_{\nu}=2 \frac{l}{L},
$$

with the length

$$
l=\sum_{\nu \neq 0}\left(\mathbf{e}_{j}^{t} \mathbf{r}_{\nu} \mathbf{l}_{\nu}^{t} \mathbf{e}_{j}\right) l_{\nu}
$$

The length $l$, which is a weighted sum of the characteristic lengths $l_{\nu}$, may be interpreted as follows: particles enter the entropy trap solely in the state $j$, i.e., the initial distribution in the variable $i$ is given by $\mathbf{e}_{j}$. This state evolves during time as $\exp (\mathbf{R} t) \mathbf{e}_{j}$. Thus, the probability to find a particle in the state $\mathbf{e}_{j}$ after time $t$ when it has initially started at $\mathbf{e}_{j}$ is

$$
p_{j}(t)=\mathbf{e}_{j}^{t} \exp (\mathbf{R} t) \mathbf{e}_{j}=p_{0 . j}+\sum_{\nu \neq 0} e^{-\lambda_{\nu} t} \mathbf{e}_{j}^{t} \mathbf{r}_{\nu} \mathbf{l}_{\nu}^{t} \mathbf{e}_{j},
$$

i.e., the nonequilibrium spectral components of this state decay with $e^{-\lambda_{\nu} t}$. The distance, particles diffuse during relaxation of the spectral component $\nu$, is $l_{\nu}$. The spectral weight factors of the initial distribution $\mathbf{e}_{j}$ is $\left(\mathbf{e}_{j}^{t} \mathbf{r}_{\nu} \mathbf{l}_{\nu}^{t} \mathbf{e}_{j}\right)$. Hence, the length $l$ may be considered as the mean length, a particle diffuses, during relaxation of $\mathbf{e}_{j}$ towards the equilibrium distribution. In other words, $l$ is the coherence length of the state $j$. The flow and first passage time may then be written as

$$
\begin{aligned}
& \tau=\tau_{0}\left(2 \frac{l}{L} e^{S}+1\right), \\
& J=J_{0} e^{S}\left(2 \frac{l}{L} e^{S}+1\right)^{-1} .
\end{aligned}
$$

The last equations demonstrate the interrelation between the entropy and the relative coherence length on the first passage time and flow. The entropy is a static quantity and independent of any transition dynamics. The specific number of particles [Eq. (65)] solely depends on this entropy. The first passage time increases with the entropy which is also intuitive since the particle may visit various states within the trap but it must return to the state $j$ before it can leave. The latter is determined by the transition dynamics, i.e., the same processes determining the coherence length. The faster this dynamics is, the smaller is the coherence length and vice versa. With increasing dynamics, the first passage time decreases and hence does the flow increase. In the limiting case of very small coherence lengths, $l / L \rightarrow 0$, one has

$$
\begin{gathered}
\tau=\frac{L^{2}}{2 D}, \\
J=\frac{c D}{L} e^{S},
\end{gathered}
$$

i.e., the first passage time is that without entropy trap and flow increases with the entropy. This may be interpreted as follows: for a vanishing coherence length one has a very rapid transition dynamics from any state to the state $j$, i.e., at the end of the entropy trap all states have almost free access to the exit and contribute to the outflow.

We will now apply the latter to the case of a discrete bounded diffusion process with equivalent transition rates between neighboring states. We assume a large number ( $I$ +1 ) of states, where $I$ is an even number allowing to locate entrance and exit states symmetrically within the states. The transition rate matrix is

$$
\mathbf{R}=D^{\prime} / \Delta^{2} \mathbf{T},
$$

where $\mathbf{T}$ is a tridiagonal matrix, with off-diagonal values 1 and diagonal values -2 except at $i= \pm I / 2$ where reflecting 
boundary conditions imply the values -1 . Here $D^{\prime} / \Delta^{2}$ is the transition rate, $D^{\prime}$ is the diffusion coefficient, and $\Delta$ is the spatial separation between the states, i.e.,

$$
W=I \Delta
$$

is the width of the entropy trap. We label the states between $-I / 2 \leqslant i \leqslant I / 2$ symmetrically around the entry and exit state's $j=0$. Symmetry of the transition matrix implies identical right- and left-sided eigenvectors, differing only by a factor. The right-sided eigenvectors are

$$
\begin{aligned}
& \mathbf{r}_{0}=\frac{1}{I+1} \mathbf{1}, \\
& \mathbf{r}_{\nu}=\frac{2}{I+1}\left(\cos \left(\frac{2 \nu \pi i}{I}\right)\right)_{i}, \\
& \mathbf{r}_{\nu}^{\prime}=\frac{2}{I+1}\left(\sin \left(\frac{(2 \nu-1) \pi i}{I}\right)\right)_{i}, \\
& \nu=1, \ldots, I / 2,
\end{aligned}
$$

where $\mathbf{1}=(1, \ldots, 1, \ldots, 1)^{t}$. For the left-sided eigenvectors we just omit the multiplicative factors to guarantee normalization.

The equilibrium probability distribution of $\mathbf{R}$ is given by the eigenvector $\mathbf{r}_{0}$, i.e., one has equal probability $(I+1)^{-1}$ in the states and since $I$ is a large number the entropy is according to Eq. (62)

$$
S=\ln (I+1) \approx \ln (I) .
$$

Since $\mathbf{e}_{0}^{t} \mathbf{r}_{\nu}^{\prime}=0$ and $\mathbf{l}_{\nu}^{\prime t} \mathbf{e}_{0}=0$ only the symmetric vectors $\mathbf{r}_{\nu}$ are relevant for the determination of flow and first passage time [see Eqs. (66) and (67)]. The eigenvalues $-\lambda_{\nu}$ of $\mathbf{R}$ are

$$
-\lambda_{\nu}=-4 \sin ^{2}\left(\frac{\nu \pi}{I}\right) \frac{D^{\prime}}{\Delta^{2}} .
$$

As mentioned above we consider the case when the characteristic lengths $l_{\nu}=\left(D / \lambda_{\nu}\right)^{1 / 2}$ are at least moderately smaller than the path length $L$, so that the relation $\tanh \left(L /\left(2 l_{\nu}\right)\right) \approx 1$ holds, and Eqs. (75) are valid. Determining $l_{1}=\left(D / \lambda_{1}\right)^{1 / 2}$ as the largest characteristic length from Eq. (81) and exploiting that $I$ is a large number, one obtains that this is equivalent to the condition that

$$
\tanh \left(\pi \frac{L}{W} \sqrt{\frac{D^{\prime}}{D}}\right) \approx 1 .
$$

The course of the hyperbolic tangent function implies that Eq. (82) is already fulfilled when $\left(L^{2} / D\right) \geqslant\left(W^{2} / D^{\prime}\right)$, i.e., the time to pass the length of the trap $\sim L^{2} / D$ is longer than that to pass the width $\sim W^{2} / D^{\prime}$.

The ratio of coherence length to length of the entropy trap is then according to Eq. (74)

$$
\frac{l}{L}=\frac{1}{I+1} \frac{W}{L} \sqrt{\frac{D}{D^{\prime}}} \sum_{\nu=1}^{I / 2} \frac{1}{I \sin (\nu \pi / I)} .
$$

For the further evaluation of the series in Eq. (83) we extract a subseries $\sum_{\nu=1}^{\nu_{l}}(I \sin (\nu \pi / I))^{-1}$, where $\nu_{l}$ is chosen small enough that

$$
\sin (\nu \pi / I) \approx \nu \pi / I,
$$

but large enough that the Euler-Mascheroni approximation holds, i.e., one has

$$
\sum_{\nu=1}^{\nu_{l}} \frac{1}{I \sin (\nu \pi / I)} \approx \frac{1}{\pi} \sum_{\nu=1}^{\nu_{l}} \frac{1}{\nu} \approx \frac{1}{\pi}\left(\ln \nu_{l}+\gamma\right),
$$

where $\gamma$ is the Euler-Mascheroni constant. For sufficient large $I$ the remaining series may be approximated by an integral, i.e.,

$$
\begin{aligned}
\sum_{\nu=\nu_{l}}^{I / 2} \frac{1}{I \sin (\nu \pi / I)} & \approx \frac{1}{\pi} \int_{\pi \nu_{l} / I}^{\pi / 2} d \xi \frac{1}{\sin (\xi)} \\
& =-\frac{1}{\pi} \ln \left(\tan \left(\pi \nu_{l} /(2 I)\right)\right) \\
& \approx-\frac{1}{\pi} \ln \left(\pi \nu_{l} /(2 I)\right),
\end{aligned}
$$

where the last approximation follows from the condition (84). The addition of the two series in Eqs. (85) and (86) then provides

$$
\sum_{\nu=1}^{I / 2} \frac{1}{I \sin (\nu \pi / I)} \approx \frac{1}{\pi}(\gamma+\ln (2 / \pi)+\ln (I)),
$$

i.e., when $I$ is large we obtain the ratio of coherence length to length of the entropy trap as [see also Eq. (80)]

$$
\frac{l}{L} \approx \frac{1}{\pi} \frac{W}{L} \sqrt{\frac{D}{D^{\prime}}} \frac{\ln (I)}{I}=\frac{1}{\pi} \frac{W}{L} \sqrt{\frac{D}{D^{\prime}}} S e^{-S}=\frac{1}{\pi} \sqrt{\frac{D}{D^{\prime}}} S e^{-S_{L}} .
$$

In the last step we exploited that $W / \Delta=I=e^{S}$, and we introduced the entropy that would be present, if the width had the value $L$, i.e., $S_{L}=\ln (L / \Delta)$. According to Eqs. (66) and (67) the first passage time and flow are

$$
\begin{aligned}
& \tau=\tau_{0}\left(\frac{2}{\pi} \sqrt{\frac{D}{D^{\prime}}} S e^{S-S_{L}}+1\right), \\
& J=J_{0} e^{S\left(\frac{2}{\pi} \sqrt{\frac{D}{D^{\prime}}} S e^{S-S_{L}}+1\right)^{-1} .}
\end{aligned}
$$

In the latter equations the first passage time and flow are directly related to the entropy and the ratio of diffusion dynamics along the path and in the additional state variable, characterized by $D / D^{\prime}$.

In Fig. 3 we considered the parameters specific particle number, first passage time, and flow as a function of the entropy difference $S-S_{L}$, i.e., the ratio of width to length of the entropy trap. Diffusion constants were taken to be equivalent, i.e., $D=D^{\prime}$. The following ranges of $S$ may be 


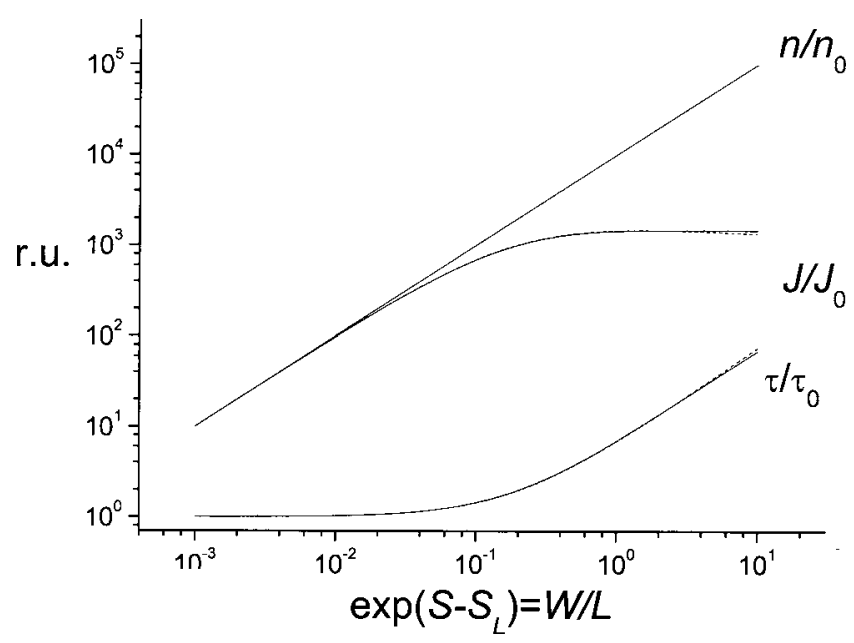

FIG. 3. Dependence of the particle number $n$, flow $J$, and first passage time $\tau$ on the entropy $S$ for the entropy trap given as an example in the text. The parameters are normalized to values in the absence of the trap $S=0$. The ratio of spatial separation between neighboring states to length is $\Delta / L$ $=10^{-4} \cdot S_{L}=\ln (L / \Delta)$ (see text). The parameter $\exp \left(S-S_{L}\right)$ is the ratio of width to length of the trap $W / L$. The lines are calculated according to Eqs. (65)-(67) (solid lines) and its approximations given by Eqs. (89) and (90) (dashed lines). The congruence is evident.

differentiated: for $S \ll S_{L}$, i.e., when the width is much smaller than the length of the trap, one has according to Eq. (89) $\tau \approx \tau_{0}$, which Fig. 3 demonstrates. However, the particle number increases with the width of the trap $\sim e^{S}$ [see Eq. (65)], i.e., flow increases by this factor as well. When $S$ is in the same magnitude as $S_{L}$, i.e., width and length are comparable, then the first passage mainly depends on $\sim e^{S-S_{L}}$, i.e., the first passage time becomes proportional to the width. Since the specific particle number scales with $e^{S}$, flow remains almost constant. In other words, an entropy trap increases flow uniformly as long as its width is considerably smaller than its length, since the specific particle number increases, whereas the first passage time is less affected. For widths beyond, the first passage time increases as the particle number does, i.e., there is no more effect on flow.

\section{SUMMARY AND CONCLUSION}

Fick's first diffusion law in its macroscopic form states that for 1D free-homogeneous diffusion between two baths flow is proportional to the concentration gradient of the baths, inverse length of the path between the baths, and the diffusion coefficient. In this paper we generalize this law to arbitrary diffusive flow of noninteracting particles in a system between two baths. Our aim was to formulate it in such a way that the concentrations of the baths acting as thermodynamic driving forces become explicit and that the conductance is solely based on a microscopic parameter which integrates information about local constraints and mobility of a particle on its way between the baths. The latter request suggests to focus on parameters characterizing the history of a particle, e.g., the first passage time, which provides information on how long a particle stays within the system.

We focused on a simplified setup, in which one bath acts as a particle source, the other as a pure absorber. Since the particles do not interact, any arbitrary presetting of concen- trations may be constructed as an additive superposition from these setups. We found that flow only depends on the concentration of the source, acting as a thermodynamic driving force, and that the conductivity is the inverse derivative of the first passage time, i.e., $J=\left(\partial_{V} \tau\right)^{-1} c$. Here $V$ is the volume of the system between the baths. Since the first passage time and hence its derivative are microscopic quantities related to a single particle, and since the particles do not interact, they are independent of the particle concentration, i.e., we have a linear diffusion law. The rather abstract microscopic parameter "inverse derivative of the first passage time" is equivalent to the intuitively better accessible ratio of the specific particle number $n$ and first passage time $\left(\partial_{V} \tau\right)^{-1}=n / \tau$. The specific particle number is the number of particles within the system normalized to the concentration of the source. For arbitrary concentrations of the two baths, $c_{1}$ and $c_{2}$, the net flow is just the difference $J=\left(n_{1,2} / \tau_{1,2}\right) c_{1}$ $-\left(n_{2,1} / \tau_{2,1}\right) c_{2}$, where the index $(1,2)$ refers to the simplified setup with bath 1 as a source with concentration $c_{1}$ and bath 2 as an absorber, and vice versa for the index $(2,1)$. In systems in which detailed balance may be achieved, the diffusive conductivities are independent of the direction of transport. When additionally there is no energy gain/loss for the particles diffusing between the baths, we can formulate a macroscopic diffusion law, similar to original Fick's law with the concentration gradient acting as a driving force for flow between the baths $J=(n / \tau)\left(c_{1}-c_{2}\right)$.

It is decisive that flow between the baths does not only depend on the first passage time, but also on the amount of particles trapped in the system $J \sim(n / \tau)$. This implies that, for example, enhancement of flow may be achieved by shortening of the first passage time, or by an increase of particle trapping. Whereas attention in the past focused mainly on the first mechanism, the latter has been neglected. We studied two scenarios of particle trapping. In the first trapping is realized by an attractive potential. Though this potential also increases the first passage time, the effect on particle trapping exceeds, which results in an increase of flow. In the second example we studied an entropy trap. This is realized by an additional degree of freedom for the particles, the state variable of which undergoes stochastic transitions with a Markovian dynamics. This system increases both the number of particles and the first passage time, but the first effect is dominating, i.e., flow increases. The increase on the number of particles trapped solely depends on the equilibrium distribution of the stochastic process in the additional state variable, i.e., it is independent of the magnitude of the transition dynamics. In contrast the first passage time strongly depends on the latter. When we extract a scaling factor from the transition dynamics of the additional state variable, e.g., a diffusion coefficient, it exhibits a reciprocal relationship with the first passage time. In the asymptotic limiting case the first passage time approaches that in the absence of the trap, and flow is purely enhanced by particle trapping.

Note that from an intuitive point of view this flow enhancement is not evident. Thinking in terms of the first passage time one would argue that an attractive potential or an entropy trap hampers particle flow by retaining the particle within the system. Only the dominating effect of particle 


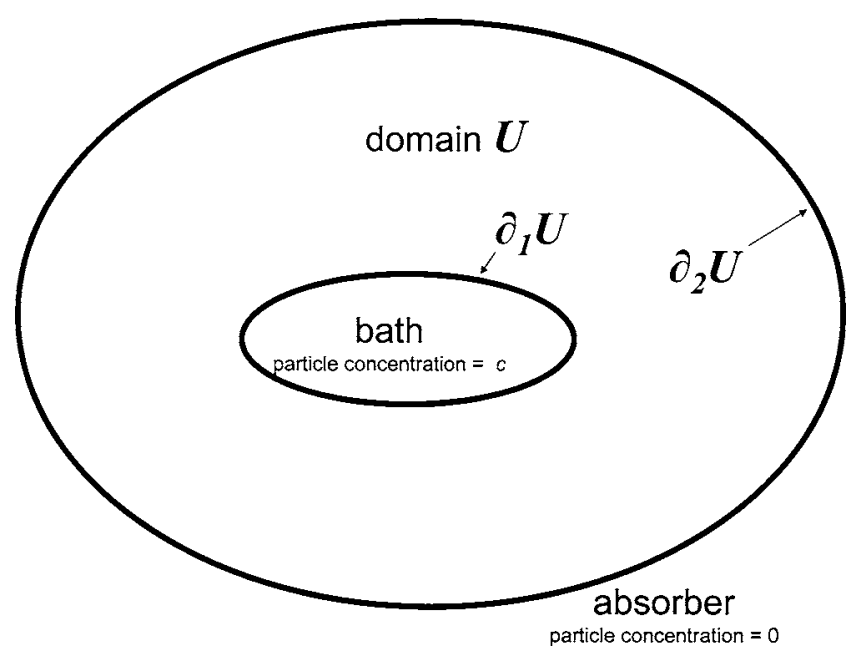

FIG. 4. Schematic of a closed domain $U$ adjacent to a bath acting as a particle source with concentration $c$, and a bath acting as a pure absorber. In the latter the particle concentration vanishes.

trapping over that on the first passage time guarantees flow enhancement. The examples of the flow enhancement by particle trapping are not only of academic interest. An example is channel transport through membranes. ${ }^{5,12}$ Here our results demonstrate that particle trapping, either by an attractive potential or an entropy trap, may facilitate particle transport. This may have important implications in biology and also in nanotechnology. Another example is derived from the fact that transport of molecules in cells is often linked to a transient chemical transition. There are numerous such processes, e.g., the transport of high energy phosphates. It is a challenge to analyze whether this transition may be considered as a particle trap facilitating the transport.

\section{APPENDIX A: FIRST PASSAGE TIME AND MACROSCOPIC STEADY-STATE PARAMETERS IN $n$ DIMENSIONS}

In this section we will relate the macroscopic steadystate parameters particle number/density and flow to the first passage time, which provides information about the history of particles in $n$ dimensions. We consider a diffusion process within some closed domain $U$ (Fig. 4). This domain is thought to have two nonconnected boundaries, e.g., $\partial U$ $=\partial_{1} U \cup \partial_{2} U$. One boundary $\left(\partial_{1} U\right)$ is adjacent to a particle bath and the other boundary $\left(\partial_{2} U\right)$ is adjacent to a bath which instantaneously absorbs particles. The Markovian diffusion dynamics is determined by a master equation, i.e., for $x \in U$ the probability density obeys

$$
\partial_{t} \rho(x, t)=\Upsilon \rho(x, t) \text {, }
$$

where $Y$ is the generator of this process. We will further restrict to processes in which Eq. (A1) has the form of a Fokker-Planck equation, i.e.,

$$
Y=\partial_{x} D(x)\left(\partial_{x}-F(x)\right),
$$

with the diffusion coefficient $D(x)$ and drift force $F(x)$. We furthermore state that the steady flow passes through the whole domain $U$.
As in the 1D case the steady-state density would remain unchanged when we introduce reflecting boundary conditions at $\partial_{1} U$ for particles within the domain $U$ and when we replace the bath by some flow source at the boundary $\partial_{1} U$ the local density of which is that of the steady-state flow, i.e., $j_{s} \mid \partial_{1} U$. The dynamics in the setup with reflecting boundary conditions at $\partial_{1} U$ is not anymore determined by Eqs. (A1) and (A2) but by

$$
\partial_{t} \rho(x, t)=\Lambda \rho(x, t)+\int_{\xi \in \partial_{1} U} d A(\xi) j_{s}(\xi) \delta(x-\xi),
$$

where $d A(\xi)$ is the $n$-dimensional surface element vector of the $n$-1D surface at position $\xi$. The new generator $\Lambda$ considers reflecting boundary conditions at $\partial_{1} U$. The integral in Eq. (A3) is just the sum over the flow sources on $\partial_{1} U$. The steady-state density derived from Eq. (A3) is identical to that of Eq. (A1). Setting Eq. (A3) to zero we obtain the steadystate density

$$
\rho_{s}(x)=-J \Lambda^{-1} w(x),
$$

where

$$
J=\int_{\xi \in \partial_{1} U} d A(\xi) j_{s}(\xi)
$$

is the overall steady-state flow. The function

$$
w(x)=J^{-1} \int_{\xi \in \partial_{1} U} d A(\xi) j_{s}(\xi) \delta(x-\xi)
$$

is the probability that a particle from the source enters the domain $U$ at position $x$. The term $-\Lambda^{-1} w(x)$ in Eq. (A4) may be rewritten in the form

$$
\begin{aligned}
-\Lambda^{-1} w(x)= & \int_{0}^{\infty} d t \int_{x^{\prime} \in U} d V\left(x^{\prime}\right)[\exp (\Lambda t)]_{x, x^{\prime}} w\left(x^{\prime}\right) \\
& -\lim _{t \rightarrow \infty} \int_{x^{\prime} \in U} d V\left(x^{\prime}\right) \Lambda^{-1}[\exp (\Lambda t)]_{x, x^{\prime}} w\left(x^{\prime}\right) \\
= & \int_{0}^{\infty} d t \int_{x^{\prime} \in U} d V\left(x^{\prime}\right)[\exp (\Lambda t)]_{x, x^{\prime}} w\left(x^{\prime}\right),
\end{aligned}
$$

where $[\exp (\Lambda t)]_{x, x^{\prime}}$ is Green's function. Since diffusion takes place within a bounded domain the eigenvalues of $\Lambda$, which are all negative, have an upper nonvanishing boundary. Hence, the last term of Eq. (A7) vanishes providing Eq. (A8). The term $\int_{x^{\prime} \in U} d V\left(x^{\prime}\right)[\exp (\Lambda t)]_{x, x^{\prime}} w\left(x^{\prime}\right)$ in Eq. (A8) is the probability to find at time $t$ at position $x$ a particle, which has left the flow source at $t=0$. Consequently, the probability $p_{U}(t)$ to find a particle in the domain $U$ is

$$
p_{U}(t)=\int_{x, x^{\prime} \in U} d V(x) d V\left(x^{\prime}\right)[\exp (\Lambda t)]_{x, x^{\prime}} w\left(x^{\prime}\right) .
$$

Hence, spatial integration of Eq. (A4) in $U$ provides 


$$
N_{U}=\int_{x \in U} d V(x) \rho_{s}(x)=J \int_{0}^{\infty} d t p_{U}(t)=J \tau_{U},
$$

where $N_{U}$ is the steady-state number of particles within $U$ and $\tau_{U}=\int_{0}^{\infty} d t p_{U}(t)$ is the mean relaxation time of the number of particles for a given initial distribution $w(x)$. Since $w(x)$ is the probability that a particle enters $U$ at position $x \in \partial_{1} U, \tau_{U}$ is, from the microscopic viewpoint, the mean live time that a particle stays within $U$, i.e., the average interval from source delivery until absorption.

The considerations above can be generalized to sourcelike domains. We define a domain $Y \subset U$, having two boundaries as "sourcelike," when the bath is within this domain, i.e., $\partial_{1} U=\partial_{1} Y$, and the whole steady-state flow $J$ passes through its outer boundary $\partial_{2} Y \subset U$. Furthermore we claim that flow is locally directed outside, i.e.,

$$
\begin{aligned}
J & =\int_{x \in \partial_{1} U} d A(x) j_{s}(x) \\
& =\int_{x^{\prime} \in \partial_{2} Y} d A\left(x^{\prime}\right) j_{s}\left(x^{\prime}\right) \text { and } j\left(x^{\prime}\right) d A\left(x^{\prime}\right)>0
\end{aligned}
$$

for $x^{\prime} \in \partial_{2} Y$.

This definition implies that the considerations above concerning the particle number and first passage time are also valid for the domain $U \backslash Y$, i.e., $\partial_{2} Y$ acts as a flow source and one has

$$
N_{U \backslash Y}=J \tau_{U \backslash Y} \cdot
$$

We now define sourcelike domains $Y_{x, \epsilon}$ for $x \in U$ as

- $Y_{x, 0}$ has $x$ in its outer boundary, i.e., $x \in \partial_{2} Y_{x, 0}$, and

- $Y_{x, \epsilon}=Y_{x, 0} \backslash S_{\epsilon}$, where $S_{\epsilon}$ is a small sphere with radius $\epsilon$ around $x$.

The existence of a domain $Y_{x, 0}$ for every $x$ follows from the fact that we assumed that the steady-state flow $j_{s}$ passes through the whole domain $U$, i.e., $j_{s}(x) \neq 0$. One has to solve the differential equation $\dot{x}=j_{s}(x)$ by $x(t)=\psi\left(x_{0}, t\right)$ with the initial condition $x(0)=\psi\left(x_{0}, 0\right)=x_{0}$. Then one has to determine the flow of the boundary $\psi\left(\partial_{1} U, t\right)$ which for a certain $t_{0}$ must reach $x$, defining $\partial_{2} Y_{x, 0}=\psi\left(\partial_{1} U, t_{0}\right)$.

Commensurate conditions for the existence of sourcelike domains $Y_{x, \epsilon}$ for sufficient small $\epsilon$ are that the steady flow density is a differentiable vector field and that the boundary $\partial_{2} Y_{x, 0}$ is a differentiable manifold within the $\epsilon$ sphere. According to Eq. (A11) we now have

$$
N_{U \backslash Y_{x, \epsilon}}-N_{U \backslash Y_{x, 0}}=J\left(\tau_{U \backslash Y_{x, \epsilon}}-\tau_{U \backslash Y_{x, 0}}\right),
$$

and since the steady-state density is

$$
\rho_{s}(x)=\lim _{\epsilon \rightarrow 0} \frac{N_{U \backslash Y_{x, 0}}-N_{U \backslash Y_{x, \epsilon}}}{V_{U \backslash Y_{x, 0}}-V_{U \backslash Y_{x, \epsilon}}},
$$

with $V$ as the volumes of the respective domains, we have

$$
\rho_{s}(x)=J \partial_{V} \tau_{U \backslash Y_{x}},
$$

or in short notation

$$
\rho_{s}(x)=J \partial_{V} \tau_{x},
$$

and especially at the boundary adjacent to the bath we have

$$
c=J \partial_{V} \tau_{x \in \partial_{1} U} .
$$

\section{APPENDIX B: STEADY-STATE PARAMETERS WITHIN THE ENTROPY TRAP}

According to Eq. (60) the steady-state condition implies for the particle density with respect to the stochastic states $\boldsymbol{\rho}=\left(\rho_{i}\right)$ that

$$
\left(\mathbf{R} / D+\partial_{x}^{2}\right) \boldsymbol{\rho}(x)=0 .
$$

This homogeneous equation is solved by function

$$
\mathbf{f}_{\nu}(x)=\mathbf{r}_{\nu} \phi_{\nu}(x),
$$

where $\mathbf{r}_{\nu}$ is a right-hand-sided eigenvector of the rate matrix $\mathbf{R}$, i.e.,

$$
\mathbf{R} / D \mathbf{r}_{\nu}=-\lambda_{\nu} / D \mathbf{r}_{\nu},
$$

with the negative eigenvalues $-\lambda_{\nu}$ of $\mathbf{R}$. Introducing the characteristic length (see main section)

$$
l_{\nu}=\sqrt{D / \lambda_{\nu}},
$$

one obtains for the scalar function $\phi_{\nu}$

$$
\partial_{x}^{2} \phi_{\nu}(x)=l_{\nu}^{-2} \phi_{\nu}(x) .
$$

The right-hand-sided eigenvectors of $\mathbf{R}$ are assumed to be normalized, i.e., with the corresponding left-hand-sided eigenvectors $\mathbf{I}_{\mu}$ they fulfill

$$
\mathbf{l}_{\mu}^{t} \mathbf{r}_{\nu}=\delta_{\mu, \nu} .
$$

The function $\mathbf{f}_{\nu}$ may be written in the form

$$
\mathbf{f}_{\nu}^{( \pm)}(x)=\mathbf{r}_{\nu} \phi_{\nu}^{( \pm)}(x),
$$

with

$$
\begin{aligned}
& \phi_{0}^{(+)}(x)=1, \\
& \phi_{0}^{(-)}(x)=-x, \\
& \phi_{\nu}^{( \pm)}(x)=e^{ \pm x / l_{\nu}} \text { for } \nu \neq 0,
\end{aligned}
$$

where we choose the index $\nu$ that $\nu=0$ corresponds to the equilibrium state of $\mathbf{R}$, i.e., $\lambda_{0}=0$. The general solution of Eq. (B1) is a linear combination of the function base given by Eqs. (B7), i.e., with coefficients $q_{\nu}^{(+/-)}$for the function $\mathbf{f}_{\nu}^{(+/-)}(x)$,

$$
\boldsymbol{\rho}(x)=\sum_{\nu} \mathbf{r}_{\nu} e^{x / l_{\nu}} q_{\nu}^{(+)}+\sum_{\nu \neq 0} \mathbf{r}_{\nu} e^{-x / l_{\nu}} q_{\nu}^{(-)}-\mathbf{r}_{0} x q_{0}^{(-)} .
$$

Insertion of $\partial_{x} \boldsymbol{\rho}(x)$ as given by Eq. (B8) into the boundary conditions (64) provides

$$
\begin{aligned}
& q_{\nu}^{(+)}-q_{\nu}^{(-)}=-l_{\nu} J / D \mathbf{l}_{\nu}^{t} \mathbf{e}_{j}, \quad \nu \neq 0, \\
& q_{0}^{(-)}=J / D \mathbf{l}_{0}^{t} \mathbf{e}_{j}=J / D,
\end{aligned}
$$

and 


$$
e^{L / l_{\nu}} q_{\nu}^{(+)}-e^{-L / l_{\nu}} q_{\nu}^{(-)}=-l_{\nu} J / D \mathbf{l}_{\nu}^{t} \mathbf{e}_{j}, \quad \nu \neq 0
$$

where we applied Eq. (B6) and that the left-hand-sided eigenvector of the equilibrium distribution is $\mathbf{l}_{0}^{t}$ $=(1, \ldots, 1 \ldots, 1) \cdot \mathbf{e}_{j}$ denotes the $j$ th unit vector in the stochastic space. Subtraction of Eq. (B9) from Eq. (B11) provides for $\nu \neq 0$

$$
q_{\nu}^{(-)}=-e^{L / l_{\nu}} q_{\nu}^{(+)}
$$

Reinsertion of the $q_{\nu}^{(-)}$into Eq. (B9) implies for $\nu \neq 0$

$$
q_{\nu}^{(+)}=l_{\nu} \frac{\mathbf{l}_{\nu}^{t} \mathbf{e}_{j}}{1+e^{L / l_{\nu}}} J / D .
$$

Equation (B8) then reads

$$
\begin{aligned}
\boldsymbol{\rho}(x)= & \left(-\frac{J}{D}\right) \sum_{\nu \neq 0} \mathbf{r}_{\nu} \mathbf{l}_{\nu}^{t} \mathbf{e}_{j} \frac{\sinh \left((x-L / 2) / l_{\nu}\right)}{\cosh \left(L /\left(2 l_{\nu}\right)\right)} l_{\nu}+\mathbf{r}_{0}\left(q_{0}^{(+)}\right. \\
& -x J / D)
\end{aligned}
$$

Insertion of $\boldsymbol{\rho}(x)$ into the boundary conditions [Eqs. (63)] gives the parameters $q_{0}^{(+)}$,

$$
q_{0}^{(+)}=\frac{J}{D}\left(L+\mathbf{e}_{j}^{t} \sum_{\nu \neq 0} \mathbf{e}_{j}^{t} \mathbf{r}_{\nu} \mathbf{l}_{\nu}^{t} \mathbf{e}_{j} \tanh \left(L /\left(2 l_{\nu}\right)\right) l_{\nu} p_{0, j}^{-1}\right),
$$

and the flow

$$
J=\frac{c D}{L}\left(\sum_{\nu \neq 0} \mathbf{e}_{j}^{t} \mathbf{r}_{\nu} \mathbf{l}_{\nu}^{t} \frac{\tanh \left(L /\left(2 l_{\nu}\right)\right)}{L /\left(2 l_{\nu}\right)}+p_{0, j}\right)^{-1},
$$

where $p_{0, j}=\mathbf{e}_{j}^{t} \mathbf{r}_{0}$ is the equilibrium probability of the state $j$.

The particle density [Eq. (B14)] is then

$$
\begin{aligned}
\boldsymbol{\rho}(x)= & -\frac{J}{D} \sum_{\nu \neq 0} \mathbf{r}_{\nu} \mathbf{l}_{\nu}^{t} \mathbf{e}_{j} \frac{\sinh \left((x-L / 2) / l_{\nu}\right)}{\cosh \left(L /\left(2 l_{\nu}\right)\right)} \\
& -\frac{J}{D} \mathbf{r}_{0}\left(L-x+\sum_{\nu \neq 0} \mathbf{e}_{j}^{t} \mathbf{r}_{\nu} \mathbf{l}_{\nu}^{t} \mathbf{e}_{j} l_{\nu} \tanh \left(L /\left(2 l_{\nu}\right)\right) p_{0, j}^{-1}\right) .
\end{aligned}
$$

The particle number is

$$
\begin{aligned}
N & =\int_{0}^{L} d x \rho(x)=\int_{0}^{L} d x \mathbf{l}_{0}^{t} \boldsymbol{\rho}(x) \\
& =-\frac{J}{D}\left(L^{2} / 2+L \sum_{\nu \neq 0} \mathbf{e}_{j}^{t} \mathbf{r}_{\nu} \mathbf{l}_{\nu}^{t} \mathbf{e}_{j} l_{\nu} \tanh \left(L /\left(2 l_{\nu}\right)\right) p_{0, j}^{-1}\right) \\
& =\frac{c L}{2} \frac{1}{p_{0, j}},
\end{aligned}
$$

where we inserted the flow from Eq. (B16). The first passage time which results from $\tau=N / J$ is [Eq. (16)]

$$
\tau=\frac{L^{2}}{2 D}\left(\sum_{\nu \neq 0} \mathbf{e}_{j}^{t} \mathbf{r}_{\nu} \mathbf{l}_{\nu}^{t} \mathbf{e}_{j} \frac{\tanh \left(L /\left(2 l_{\nu}\right)\right)}{L /\left(2 l_{\nu}\right)} p_{0, j}^{-1}+1\right) .
$$

Since $p_{0, j}=e^{-S}$ one obtains Eqs. (65)-(67) for the particle number, first passage time, and flow of the main section.

${ }^{1}$ A. Fick, Ann. Phys. 94, 59 (1855).

${ }^{2}$ This implies that self-interaction of particles is neglected, which is always assumed throughout the paper.

${ }^{3}$ S. Hardt, Bull. Math. Biol. 43, 89 (1981).

${ }^{4}$ A. M. Berezhkovskii, M. A. Pustovoit, and S. M. Bezrukov, J. Chem. Phys. 22, 9952 (2002).

${ }^{5}$ E. M. Nestorovich, C. Danelon, M. Winterhalter, and S. M. Bezrukov, Proc. Natl. Acad. Sci. U.S.A. 15, 9789 (2002).

${ }^{6}$ For arbitrary concentrations in the baths one has bidirectional transport which is just a superposition of our unidirectional transport, as long as particles are noninteracting.

${ }^{7}$ Since we do not consider temperature dependence, we set $k T=1$ in the Fokker-Planck equation, i.e., we write $F(x)$ instead of $F(x) / k T$. This is done throughout the manuscript and also refers to potentials.

${ }^{8}$ This is always fulfilled in the $1 \mathrm{D}$ case as long as circular topologies of the interval are excluded.

${ }^{9}$ K. Schulten, Z. Schulten, and A. Szabo, J. Chem. Phys. 74, 4426 (1981).

${ }^{10}$ W. Nadler and K. Schulten, J. Chem. Phys. 82, 151 (1984).

${ }^{11}$ H. A. Kramers, Physica (Amsterdam) 7, 284 (1940).

${ }^{12}$ L. Dagdug, A. Berezhkovskiia, S. M. Bezrukov, and H. Weiss, J. Chem. Phys. 118, 2367 (2003). 